\title{
MODELO DE ANÁLISIS PROPOSICIONAL (MAP) PARA EL CONCEPTO EVOLUCIÓN EN ESTUDIANTES DEL BACHILLERATO TECNÓLÓGICO
}

\author{
Sergio R. Torres Ochoa \\ Universidad Michoacana de San Nicolás de Hidalgo, México \\ J. Francisco Chávez Aguilar \\ Centro de Estudios Tecnológicos, Industrial y de Servicios No. 120, México \\ Alicia Romero Alegre \\ Centro de Bachillerato Tecnológico Industrial y de Servicios No. 149, México
}

\section{DEFINICIÓN DEL PROBLEMA}

Hemos observado que un gran porcentaje de alumnos en los planteles estudiados, han reprobado la asignatura de Biología (25.35\% en el CBTis $149^{1}$ y $27.15 \%$ en el CETis $120^{2}$ en los tres últimos años); tal parece que dicha materia representa un reto de elevado grado de dificultad.

Tratando de analizar los motivos, nos encontramos con una serie de situaciones predisponentes, en los alumnos, en los docentes, en las condiciones de la escuela y en el currículum.

Los alumnos son los principales actores afectados por los programas de educación debido, entre otras cosas, a la falta de capacitación y actualización de los docentes, a su propio desinterés y a la falta de motivación por el estudio.

Después de haber realizado una evaluación tanto de los factores sociales como de los personales de la problemática anterior se seleccionó, como objeto de estudio relevante, la dificultad que tienen los estudiantes en comprender la evolución biológica como principal componente epistemológico para la asimilación básica del conocimiento sobre la Biología. Consideramos que el factor cognitivo posee un fundamental interés para la intención rigurosa de conocer las predisposiciones y potencialidad de asimilar conceptos, en especial de una ciencia como la Biología.

Para que los alumnos, objeto del estudio, sean capaces de aplicar los conceptos a una variedad de contextos necesitan no sólo ser capaces de definirlos, sino también de relacionarlos entre sí, ya que, como se indica en el programa de estudios, “... su función es establecer los procesos y procedimientos que permitan una correlación de conocimientos, técnicas y actitudes que garanticen la incorporación significativa y operacional del campo de la Biología al perfil del bachiller".

Necesitan además diferenciar el concepto de evolución de otros conceptos relacionados, porque "...el tratamiento propuesto en el programa tiene un enfoque evolutivo, lo cual permitirá que los alumnos comprendan que el universo no es estático, sino que va cambiando a través del tiempo, además de que toma como base los niveles de organización de la materia y la energía, yendo de lo simple a lo complejo. Esto permite al alumno la opción de que, conforme el programa se va desarrollando, pueda integrar los temas del curso en torno a este concepto general evolutivo".

\footnotetext{
${ }^{1}$ Centro de Bachillerato Tecnológico Industrial y de Servicios No 149, México.

${ }^{2}$ Centro de Estudios Tecnológicos Industrial y de Servicios No 120, México.
} 
La falta de atención sobre el razonamiento cualitativo y el conocimiento conceptual dienta los enfoques de repetición y memorización. En este sentido, el concepto de enseñanza de la ciencia que aquí se asume, o sea, la forma de introducirse en el conocimiento científico,

puede establecerse como el equilibrio de asimilación de conocimientos previos y la búsqueda individual de nuevos conocimientos.

Desde el punto de vista específico del problema que nos ocupa es muy importante para el alumno que comprenda y pueda construir conocimientos a partir de las organizaciones de conceptos y principios generales de la Biología. Con el objeto de que interprete y dé solución a los planteamientos que se le presentan durante sus estudios posteriores; que aprenda a observar lo que le rodea; que reconozca los efectos de la intervención humana; que se familiarice con los fenómenos cotidianos, con la tecnología de uso diario y sus interacciones políticas y sociales.

En este trabajo se considera el aprendizaje significativo del concepto evolución desde una perspectiva constructivista. Su intención es llegar al pensamiento del alumno más que al hecho del aprendizaje memorista de una formula, procedimiento o definición. Se pretende que el aprendizaje se convierta en un fenómeno complejo que sobrepase las simples asociaciones memoristas, es decir, una organización activa de los conceptos y esquemas que posea el alumno en su estructura cognitiva.

\section{Objetivo general}

Evaluar, a partir del análisis discursivo, las diferencias entre las estructuras cognitivas del estudiante de Biología y las de los docentes con respecto al concepto Evolución.

\section{Objetivos específicos}

- Analizar las estructuras de conocimiento representadas en declaraciones proposicionales contenidas en el discurso de los alumnos.

- Determinar la correspondencia lógico-conceptual entre la organización conceptual del profesor y la del alumno.

- Calcular la porción común de las organizaciones conceptuales entre el profesor y el alumno.

\section{FUNDAMENTOS PSICOPEDAGÓGICOS DEL APRENDIZAJE}

Vygotsky nos dará todo su apoyo con sus aportes al constructivismo de orientación sociocultural. Donde el lenguaje aparece como un instrumento de mediación cultural capaz de activar y regular el comportamiento, tras ser interiorizado, para que el alumno logre el aprendizaje. Sin olvidar las experiencias previas del mundo real que los alumnos poseen y que éstas experiencias las tienen organizadas de una forma particular tal que les permite explicar a su modo los hechos reales. Estos son aspectos que intentamos valorar con el manejo de un modelo de análisis que pueda ser útil a investigadores y docentes en conocer el estado y condiciones de asimilación conceptual de los alumnos con respecto a conceptos eje o claves dentro de la estructura cognitiva referida a alguna materia científica (Mool, 1993). 
En el caso específico de la enseñanza de la evolución biológica, en nuestras escuelas, generalmente ésta es inefectiva debido a que el énfasis que se le da es muy pobre, lo que ocasiona que el alumno no pueda integrar estos conocimientos de manera significativa.

Ausubel establece que el aprendizaje significativo sucede cuando éste puede relacionarse de modo no arbitrario y sustancial con lo que el alumno ya sabe. De tal manera que si el alumno no tiene un conocimiento previo sobre determinado contenido, éste contenido carecerá de significado para él (Ausbel, 1983). A este hecho se eslabona el contexto social del estudiante, es decir, su interacción con el medio social y cultural en que se desenvuelve.

Piaget explica cómo aprenden los alumnos y la importancia de organizar actividades adecuadas para que aprendan. Sobre todo para que aprendan por ellos mismos y sean capaces de aplicar el conocimiento a situaciones nuevas; tenemos entonces que ocuparnos de cómo construyen su inteligencia. Los alumnos no sólo forman conocimientos concretos sino que adquieren sistemas para recibir información y transformarla. Más que los conocimientos concretos que posee un alumno, lo que le capacita para aprender son las formas de abordar los problemas que enfrenta cotidianamente (Fraisse, 1973).

Para entender los mecanismos de aprendizaje, se requiere el conocimiento del estado en que se encuentra el alumno cognoscente. Es decir, cómo va a recibir ese nuevo conocimiento con base en el bagaje de conocimientos previos que establezcan las relaciones pertinentes entre sus haberes, saberes y el nuevo conocimiento. Por lo tanto, resulta importante conocer los distintos estadios por los que pasa el alumno en su desarrollo mental.

Coll, parte del principio de que todo acto educativo implica una referencia obligada a un proceso de adquisición de saberes Entendiendo por "saberes", tanto los contenidos específicos de las distintas áreas del aprendizaje escolar como las destrezas, normas, valores, actitudes e intereses que vinculan los sistemas educativos (Coll, 1998). Propone llamar "esquemas de conocimiento" a las representaciones que una persona posee en un momento determinado de su historia sobre una parcela de la realidad Un esquema de conocimiento puede ser más o menos rico en información y detalles; poseer un grado de organización y de coherencia interna, variable y ser más o menos válido, es decir, más o menos adecuado a la realidad. Un esquema de conocimiento comporta esquemas de acción y esquemas representativos en el sentido piagetano; los diferentes elementos que lo constituyen presentan una organización interna que puede ir desde la simple yuxtaposición hasta estructuras ordinales y jerárquicas operatorias.

\section{ESTRATEGIA METODOLÓGICA}

Consiste en la adquisición de información, tanto teórica como empírica, utilizando la metodología propuesta por el Modelo de Análisis Proposicional (MAP) (Campos, Cortes y Gaspar,1999; Campos y Gaspar, 1996; Campos, Gaspar y Alucema, 2000). Este modelo se ha diseñado para identificar las ideas principales de una organización conceptual de acuerdo a su contenido lógico y conceptual, mediante el análisis de proposiciones.

En este modelo se define a la proposición como una declaración temática especifica y dependiente de contexto, la cual tiene las siguientes características: 
- Está formado por dos o más conceptos y una relación lógica por lo menos.

- Pertenece o es en sí misma una zona de conocimiento o formación temática, y

- Comunica significado contextual.

La mayoría de las proposiciones contienen más de dos conceptos y una relación debido a la necesidad lingüística de comunicar una idea o formación temática. Para los propósitos de nuestro análisis, un concepto se define como un conjunto de palabras (generalmente una), que nombra o define un objeto (abstracto o concreto), un evento o proceso; se reconocen en los sustantivos del discurso. Una relación lógica se define como un conjunto de palabras, generalmente una, que describe una acción .

Dado que el discurso científico es una representación de la realidad epistemológica, metodológica y socialmente aceptada como válida, las organizaciones conceptuales de carácter científico son coherentes y significativas cuando se construyen mediante métodos rigurosos e información constatable.

Así el MAP es un método de análisis de discurso para estudiar la estructura de cualquier texto, sin importar en qué clase se pueda ubicar (Campos, Ruiz y Alucema, 1996; Alucema, 1996; Alucema, 2001).

Finalmente el MAP consta de los siguientes rubros a desarrollar:

- Obtención de información

- Análisis del discurso

- Análisis de correspondencia

- Análisis cualitativo de la correspondencia

- -Análisis cuantitativo de la correspondencia

- -Índices

- Clasificación de la organización conceptual

\section{ANÁLISIS CUANTITATIVO DE LA CORRESPONDENCIA}

La mayoría de los discursos, aun los científicos, incluyen componentes semánticos no necesarios o inadecuados que redicen su claridad.

Esta situación es más evidente en el caso del aprendizaje. Cuando se esta adquiriendo conocimiento nuevo, la preescisión no se aprende inmediatamente.

Así una proposición adecuada de conceptos y relaciones lógicas producirá un texto con la densidad suficiente para ser entendido.

La densidad por lo tanto se define como la proporción del numero de conceptos, C, respecto al número de las relaciones lógicas, $\mathbf{R}$ : por lo tanto d= C/R (Campos, 1996).

ÍNDICES (Campos, Cortés y Gaspar, 1999; Campos y Gaspar, 1996; Campos, Gaspar y Alucema, 2000). 
Correspondencia conceptual: se refiere a la proporción de conceptos en correspondencia $\left(\mathbf{C}_{\mathbf{T S}}\right)$, respecto al total de conceptos en la organización conceptual criterio $\left(\mathbf{C}_{\mathbf{T}}\right)$, y se denota como: $\mathbf{c c}=\mathbf{C}_{\mathbf{T S}} / \mathbf{C}_{\mathbf{T}}$.

Correspondencia relacional: proporción del número de relaciones lógicas que el estudiante usa en el conjunto de conceptos en correspondencia ( $\left.\mathbf{R}_{\mathbf{S T C}}\right)$, respecto del total de relaciones lógicas con respecto al criterio $\left(\mathbf{R}_{\mathrm{TC}}\right)$, y se denota como: $\mathbf{c r}=\mathbf{R}_{\mathbf{S T C}} / \mathbf{R}_{\mathrm{TC}}$

Correspondencia en el núcleo conceptual: proporción del número de conceptos de la organización del estudiante $\left(\mathbf{C}_{\mathbf{S T C}}\right.$ ), respecto a los que se encuentran en el núcleo conceptual del criterio $\left(\mathbf{C}_{\mathrm{TC}}\right)$, y se denota como: $\mathbf{c}=\mathbf{C}_{\mathrm{STc}} / \mathbf{C}_{\mathrm{TC}}$

Calidad en conceptos y relaciones lógicas: los conceptos y las relaciones lógicas en una organización conceptual tiene significado solamente cuando se presentan en conjunto por las razones de estructuración discursiva. Significa, por tanto, identificar la zona de intersección de dos diferentes organizaciones conceptuales, la del estudiante y la del criterio, dicha intersección se denota como: $\mathbf{q}=$ (cc . cr)

Calidad general del discurso: además de la calidad en correspondencia en masa informativa y relacional, el núcleo conceptual es tan importante que su asimilación es factor de calidad en la organización conceptual del estudiante. Al agregarlo al factor $\mathbf{q}$ se tiene un índice general de calidad con base en la correspondencia en precisión y lo central o básico $(\mathbf{q}+\mathbf{c})$ de una zona de conocimiento determinada. Así mismo es necesario involucrar el valor de la densidad, la cual se encuentra en el plano sintáctico organizador del discurso, como ponderación de la calidad en precisión, que está en el plano semántico. Este índice general se denota como: $\mathbf{Q}=(\mathbf{q}+\mathbf{c}) / \mathbf{d}$ que significa que se ha asimilado masa informativa y relacional en conjunto con aspectos básicos o centrales de acuerdo con una estructura sintáctica.

\section{CLASIFICACIÓN DE LA ORGANIZACIÓN CONCEPTUAL}

Hipótesis de valores mínimos de correspondencia y densidad: La correspondencia varía entre ninguna $(c c=c r=c=0)$ y total $(c c=c r=c=1)$, mientras que la densidad puede ser muy fuerte $(d>2)$ o muy débil $(d=0)$, que represente una compleja combinación de niveles de asimilación en precisión en masa informativa, relacional, básica, y la forma de expresarla, de una zona determinada de conocimiento.

De acuerdo a lo anterior se plantean las siguientes hipótesis. Sobre los valores de variación de los índices mencionados para la clasificación de organizaciones conceptuales:

- Hipótesis de asimilación de conceptos: De acuerdo con los elementos teóricos anteriores, el estudiante asimila sólo una porción de los componentes conceptuales de una zona de conocimiento en condiciones regulares de aula, por lo tanto se plantea que esta porción es igual o menor a la mitad de esta zona en esas condiciones, es decir $\mathbf{C}_{\mathrm{TS}} \leq \mathbf{0 . 5} \mathbf{C}_{\mathbf{T}}$

- Hipótesis de precisión en asimilación de relaciones lógicas que conectan conceptos en correspondencia: la asimilación relacional implica un nivel de precisión muy alto en la masa conceptual en correspondencia. Debido a que ésta puede ser equivalente o alusiva, se puede reducir la precisión en las conexiones lógicas entre conceptos. Por ello, lo más probable es que se 
asimile sólo una parte de las relaciones lógicas entre conceptos en correspondencia $\left(R_{S T C}<R_{T C}\right.$ ) por lo que se plantea $\mathbf{R}_{\mathrm{STC}} \leq \mathbf{0 . 5} \mathbf{R}_{\mathrm{TC}}$

- Hipótesis de precisión en asimilación de los conceptos centrales en una zona determinada de conocimiento: dado que no se asimila toda una zona de conocimientos, interesa saber si sus componentes fundamentales se asimilan. Se supone que en este aspecto hay una situación igual a las anteriores. De ser así, se asimila el núcleo conceptual en forma parcial $\left(\mathrm{C}_{\mathrm{STc}}<\mathrm{C}_{\mathrm{TC}_{\mathrm{C}}}\right)$, por lo tanto; $\mathrm{C}_{\mathrm{STc}} \leq 0.5 \mathrm{C}_{\mathrm{Tc}}$.

- Hipótesis de necesidad lingüística en la generación de discurso: un nivel dado de coherencia en un discurso amplio es difícil de mantener, por lo que la densidad varía. Se plantea que la densidad varia en un rango $1 \leq d \leq 2$, sin requerir componentes redundantes o no pertinentes.

Rangos para la clasificación de la organización conceptual:

- Marcos conceptuales: de acuerdo a los elementos teóricos y las hipótesis anteriores, un estudiante ha asimilado conocimiento sustancial en clase cuando muestra por lo menos la mitad de una combinación de información requerida, formas de estructurarla lógicamente y su texto presenta una densidad de $d \leq 1.38$

Así, $c c=c r=c \geq 0.5$ y d $\leq 1.38, Q=\left[\left(c c^{*} c r\right)+c\right] / d \geq\left[\left(0.5^{*} 0.5\right)+0.5\right] / 1.38=0.543$. De esta manera, el índice $Q \geq 0.543$ representa una organización conceptual fuerte. Debido a que una correspondencia perfecta $(\mathrm{cc}=\mathrm{cr}=1)$ y alta densidad $(\mathrm{d}=1)$ producen un valor de $\mathrm{Q}=2$, podríamos esperar que el rango para marcos conceptuales sea de $0.543 \leq Q \leq 2$.

Este rango representa una organización conceptual con un buen número de conceptos científicos, entre ellos un numero razonable de conceptos básicos de una zona de conocimiento determinada, algunas relaciones lógicas apropiadas y un equilibrio adecuado de esos componentes

- Marcos referenciales, las organizaciones conceptuales de este tipo representan una correspondencia y densidad menores cuando $0.25 \leq \mathrm{cc}=\mathrm{cr}=\mathrm{c}<0.5$, con lo que $0.0625 \leq \mathrm{q}<$, mientras que $1.38<\mathrm{d} \leq 2$. Los valores que producen un valor más alto de $Q$ en este rango serian $\mathrm{cc}=\mathrm{cr}=\mathrm{c} \approx 0.499 \mathrm{yd} \approx 1.381$, mientras que los más bajos serian $\mathrm{cc}=\mathrm{cr}=\mathrm{c}=0.25 \mathrm{y} \mathrm{d}=2$, por lo que el rango para marcos referenciales es de $0.156 \leq Q<0.543$

Esto significa que una porción razonable de conceptos, entre ellos algunos centrales se han asimilado, junto con algunas formas apropiadas de conectarlos en el marco de una estructura sintáctica aceptable de conceptos y relaciones

- Marcos nocionales: este tipo de organizaciones es el más pobre. Los valores que la representan son $\mathrm{cc}=\mathrm{cr}=\mathrm{c}<0.25 \mathrm{y} \mathrm{d}>2$. El valor más alto de $Q \approx 0.155$ se da cuando los valores de estos índices se aproxima a los limites del rango superior ( $c c=c r=c \approx 0.249$ y $d \approx 2.01$ ). Por otro lado, aparte del caso en que los valores de correspondencia son igual a cero y por tanto $Q=0$, un valor muy bajo es $\mathrm{Q}=0.044$ cuando $\mathrm{cc}=\mathrm{cr}=\mathrm{c} \approx 0 \mathrm{y} \mathrm{d}=2$. Por lo tanto el rango para marcos nocionales es de $0 \leq Q<0.156$. 


\section{OBTENCIÓN DE INFORMACIÓN DE LOS ESTUDIANTES}

Se aplicó un examen de una pregunta sobre evolución biológica a los alumnos que cursan la materia de Biología en el segundo semestre de las especialidades de Laboratorista Clínico y Puericultura (área Químico-Biológicas) en el Centro de Bachillerato Tecnológico Industrial y de Servicios No 149 (CBTis 149); igualmente a alumnos del Centro de Estudios Tecnológico Industrial y de Servicios No 120 (CETis 120), de las especialidades: Administración de Empresas, Computación y Electrónica (área EconómicoAdministrativa). Ambos planteles ubicados en la ciudad de Morelia, Estado de Michoacán, México. El tema Evolución Biológica se encuentra dentro de la Cuarta Unidad (Diversidad, consecuencia de la Evolución) del programa de estudios de la materia de Biología. La prueba de obtención de información se aplicó antes y después de haber abordado dicha unidad durante el semestre escolar. La pregunta fue elaborada a partir de la respuesta criterio o argumentación extraía de los profesores que imparten la materia en cuestión. El tiempo asignado para responder la prueba fue de 20 minutos.

La intención fue que el estudiante respondiera de manera argumentativa, es decir, que mostrara explicaciones y argumentaciones sobre el contenido conceptual y las relaciones lógicas en que está basado su conocimiento del concepto (evolución biológica). El nivel de demanda cognitiva se establece en el de argumentación: producción de declaraciones que, además, deben incluir definiciones que se desarrollen mediante discurso explicativo. Con esto se logra que los estudiantes hagan explícitos sus conceptos y las formas de conectarlos. Por tanto, las respuestas de los estudiantes fueron de tipo ensayo con conceptos y relaciones lógicas.

De acuerdo a lo anterior, al momento de aplicar el cuestionario se les pidió a los alumnos que contestaran la pregunta planteada, a manera de discurso. Esto es, que se trataba de una pregunta abierta en la cual ellos podrían explicar lo solicitado de manera libre y con sus propias palabras.

Cabe aclarar que antes de aplicar el cuestionario a los grupos sujetos a investigación se llevó a cabo la validación de la pregunta. Esto se hizo aplicando el cuestionario a un grupo de alumnos (prueba piloto) que no formaron parte de la muestra elegida con objeto de saber si la pregunta planteada es clara y no causa confusión.

\section{OBTENCIÓN DE LA INFORMACIÓN DEL CRITERIO}

Los profesores que imparten clase a los grupos seleccionados también respondieron el examen aplicado a su grupo. Lo anterior se hizo con el propósito de contar con un criterio de comparación para el análisis de la estructuras conceptuales de los estudiantes. Las respuestas de los profesores se tomaron como fuente criterio por las siguientes razones.

- a) son los responsables directos de que los alumnos enfrenten en el aula el concepto Evolución Biológica,

- b) son quienes traducen conocimiento científico biológico a discurso enseñable en el CBTis y CETis,

- c) son los productores del discurso especifico al que están expuestos los estudiantes en el aula; 
- d) son los intermediarios principales entre su discurso y cualquier conocimiento que obtengan los estudiantes, en clase o por su cuenta, mediante la selección de lecturas, asignación de tareas, coordinación de la discusión en clase y la interacción y de la evaluación.

En seguida se analizaron las respuestas proporcionadas por los profesores (seis):

- Se identificaron los conceptos comunes y que aparecían en todos los cuestionarios de los maestros; adaptándolos a una misma terminología.

- Los conceptos que no fueron comunes, pero que fueron considerados importantes, también se incluyeron dentro del cuerpo de la respuesta criterio. Esto se hizo con base en la consulta de la literatura especializada y actualizada sobre el tema.

- Aquellos conceptos que estuvieron fuera del contexto de interés para el estudio a realizar fueron eliminados.

De manera tal que del análisis de estos ensayos-respuesta se obtuvo el criterio. Ello nos permitió comparar el conocimiento recibido por los alumnos, el que realmente asimilan e integran a sus estructuras conceptuales, y considerando los siguientes factores:

- Son distintos profesores para cada grupo de alumnos, los cuales llevan un mismo programa de estudios, en ambas escuelas.

- Que las especialidades que se eligieron para realizar el estudio tres pertenecen al área de Químico-Biológicas y las otras tres al área de Económico-Administrativas; lo cual es un indicador de que no necesariamente los alumnos de estos grupos tendrán a la Biología como campo profesional

- Las especialidades pertenecen a dos planteles diferentes, pero del mismo subsistema.

- Las muestras que se analizaron se eligieron aleatoriamente. Es decir, de un universo formado por seis grupos de aproximadamente 40 alumnos (240 en total), se extrajo una muestra de 50 alumnos elegidos al azar de la siguiente manera: de acuerdo al orden que tenían las pruebas al momento de la entrega por parte de los alumnos se tomaron quintas: el 1, 5, 10,15, etcétera.

\section{ANÁLISIS DE RESULTADOS}

\section{Análisis de la calidad del discurso del estudiante}

Se presenta aquí el análisis cualitativo de 12 alumnos de la muestra en estudio, 6 de cada uno de los planteles y el criterio establecido es el valor de $Q$ (calidad general del discurso), de manera que se analizan los dos valores más altos de $Q$, los dos más bajos y los dos que se acerquen a la media obtenida por plantel.

De los resultados obtenidos en el CBTis 149 con la $Q$ más alta, en la postprueba, tenemos lo siguiente:

Los alumnos que obtuvieron una $Q$ alta son el alumno $A 14(Q=1.5)$ y $A 12(Q=1.49)$, se encuentran dentro del rango de Marco Referencial de la calidad del discurso. En lo referente al alumno A14 presenta dos proposiciones, lo cual no ocurre con el alumno A12. Ambos incluyen dentro de su discurso los 2 núcleos conceptuales del criterio; el núcleo cambio lo manejan de forma idéntica. Pero en el caso de la 
evolución biológica, que es el otro núcleo, el alumno A12 lo hace utilizando un concepto equivalente y el A14 un concepto alusivo. El numero de conceptos que el A14 incluye dentro de su discurso y que corresponden al criterio son 6 y 3 relaciones lógicas. El A12 utiliza 5 conceptos del criterio y también 3 relaciones lógicas. Con respecto a estas últimas, dos de ellas son iguales para los dos alumnos.

- Los alumnos presentan por lo general, una estructura sintáctica poco clara, aspecto que se ve reflejado en los valores de densidad que se encuentran por debajo de la proporción esperada.

- Las relaciones lógicas que se utilizan para conectar los conceptos en la mayoría de ellos son innecesarias o utilizan formas redundantes.

- La demanda cognoscitiva de la organización del criterio es muy alta, amplia y densa, con una fuerte carga de relaciones explicativas. Las respuestas de los alumnos generalmente son débiles y si la demanda cognitiva es fuerte probablemente tiene que ver con los índices de reprobación y deserción en la materia de Biología.

- Los alumnos presentan sólo una aproximación descriptiva en el estudio de la evolución, concepto que permea todos los conceptos biológicos. Lo cual genera problemas epistemológicos y cognoscitivos que tienen que ver con el hecho de que los alumnos tengan información incompleta y no asimilen el nivel fino de la explicación científica y los mecanismos lógicos que permiten llegar al conocimiento Biológico.

- Los niveles de precisión en el uso de conceptos científicos que se presentan en la mayoría de los casos son de equivalencia y alusivo. Dado que las respuestas se encuentran en una zona de conocimiento y un contexto escolar y social específico, los alumnos utilizan términos de diversa índole al referirse a conceptos que en el conocimiento científico son precisos.

\section{Análisis cuantitativo}

\begin{tabular}{|c|c|c|c|c|c|c|c|c|c|c|c|c|c|c|c|}
\hline \multicolumn{10}{|c|}{ Resultados del análisis de correspondencia global preprueba } \\
\hline ALUMNO & DENSIDAD & C.I & C.E & C.A & CC & R.I & R.E & R.A & CR & CNCI & CNCE & CNCA & C & q & Q \\
\hline A1 & 1.3 & 1 & 2 & & 0.187 & & 2 & & 0.142 & 1 & & & 0.5 & 0.03 & 0.406 \\
\hline A2 & 1.22 & & 3 & & 1.89 & 1 & 3 & & 0.285 & & 1 & & 0.5 & 0.05 & 0.453 \\
\hline A3 & 1 & 1 & 3 & & 0.25 & 1 & 1 & & 0.142 & & 2 & & 1 & 0.04 & 1.035 \\
\hline A4 & 1.1 & 1 & 3 & 1 & 0.312 & 2 & & & 0.142 & & 1 & 1 & 1 & 0.04 & 0.949 \\
\hline A5 & 1.3 & 2 & 2 & & 0.25 & & & 1 & 0.071 & & 1 & & 0.5 & 0.02 & 0.398 \\
\hline A6 & 1 & 1 & 3 & & 0.25 & 2 & & 2 & 0.28 & & 2 & & 1 & 0.07 & 1.07 \\
\hline A7 & 1.2 & & 2 & 3 & 0.312 & 1 & & & 0.071 & & 1 & & 0.5 & 0.02 & 0.435 \\
\hline A8 & 1.5 & & 2 & 1 & 0.187 & & & 1 & 0.071 & & 1 & & 0.5 & 0.01 & 0.342 \\
\hline A9 & 1.2 & 4 & & & 0.25 & & 1 & & 0.071 & 2 & & & 1 & 0.02 & 0.848 \\
\hline A10 & 4 & 2 & & & 0.125 & & & 1 & 0.071 & & 1 & & 0.5 & 0.09 & 0.127 \\
\hline A11 & 1.3 & 2 & & 1 & 0.187 & & 1 & & 0.071 & 1 & & 1 & 1 & 0.01 & 0.779 \\
\hline A12 & 1.16 & & 3 & & 0.187 & & & 1 & 0.071 & & 1 & & 0.5 & 0.01 & 0.442 \\
\hline A13 & 1.3 & & & & 0.25 & & & & 0.214 & & & & 0.5 & 0.05 & 0.425 \\
\hline
\end{tabular}




\begin{tabular}{|c|c|c|c|c|c|c|c|c|c|c|c|c|c|c|c|}
\hline A14 & 1.25 & 2 & 1 & & 0.187 & 2 & & & 0.214 & 1 & & & 0.5 & 0.04 & 0.432 \\
\hline A15 & 1.75 & 1 & 2 & & 0.187 & 1 & 1 & & 0.142 & & 2 & & 1 & 0.03 & 0.586 \\
\hline A16 & 1.5 & & 2 & & 0.125 & & & & 0 & & 1 & & 0.5 & 0 & 0.333 \\
\hline $\mathrm{A} 17$ & 1.2 & & 2 & & 0.125 & 1 & 1 & & 0.142 & & 1 & & 0.5 & 0.02 & 0.421 \\
\hline A18 & 1.37 & 3 & 2 & & 0.187 & 2 & 1 & & 0.214 & 2 & & & 1 & 0.04 & 0.759 \\
\hline A19 & 1.16 & 1 & 2 & & 0.187 & & 1 & & 0.071 & & 1 & & 0.5 & 0.01 & 0.442 \\
\hline A20 & 1.25 & 1 & 2 & & 0.187 & 2 & 1 & & 0.214 & 1 & & & 0.5 & 0.04 & 0.432 \\
\hline A21 & 1.3 & 1 & 1 & 1 & 0.187 & 1 & & & 0.071 & 1 & & & 0.5 & 0.01 & 0.394 \\
\hline A22 & 1.2 & 1 & 2 & & 0.187 & 1 & & 1 & 0.142 & & 2 & & 1 & 0.03 & 0.855 \\
\hline A23 & 1.12 & 2 & 1 & 1 & 0.312 & 2 & 1 & & 0.214 & 1 & & 1 & 1 & 0.07 & 0.952 \\
\hline A24 & 0.75 & 2 & & & 0.125 & & 1 & 2 & 0.214 & & 1 & & 0.5 & 0.03 & 0.702 \\
\hline A25 & 1.5 & 1 & 1 & 1 & 0.187 & 1 & & 1 & 0.142 & & 1 & & 0.5 & 0.03 & 0.351 \\
\hline A26 & 1.16 & 1 & & & 0.062 & 1 & & & 0.071 & & & & 0 & 0 & 0.003 \\
\hline A27 & 1.5 & 1 & 1 & 1 & 0.125 & & & & 0 & & & & 0 & 0 & 0 \\
\hline A28 & 1.25 & & 1 & 3 & 0.25 & & & & 0 & & & & 0 & 0 & 0 \\
\hline A29 & 1.6 & 1 & 3 & & 0.25 & 2 & & & 0.142 & & & & 0 & 0.04 & 0.022 \\
\hline A30 & 1.5 & 2 & 1 & & 0.125 & 1 & & & 0.071 & & & & 0 & 0.01 & 0.005 \\
\hline A31 & 3 & & 2 & 2 & 0.25 & & 1 & & 0.071 & & & & 0 & 0.02 & 0.005 \\
\hline A32 & 1 & 2 & 1 & & 0.187 & 1 & 1 & & 0.142 & & & & 0 & 0.03 & 0.026 \\
\hline A33 & 1 & 1 & 2 & 1 & 0.25 & 1 & 1 & & 0.142 & & & & 0 & 0.04 & 0.035 \\
\hline A34 & 2 & 1 & 2 & & 0.187 & & 1 & & 0.071 & & & & 0 & 0.01 & 0.006 \\
\hline A35 & 1.5 & 1 & 3 & & 0.25 & & 2 & & 0.214 & & & & 0 & 0.01 & 0.003 \\
\hline A36 & 1.5 & & 1 & 1 & 0.125 & & 1 & & 0.071 & & & & 0 & 0.01 & 0.005 \\
\hline A37 & 2.5 & 1 & 1 & & 0.125 & & 1 & & 0.071 & & & & 0 & 0.01 & 0.003 \\
\hline A38 & 1.5 & 1 & 2 & 1 & 0.25 & 1 & & & 0.071 & & & & 0 & 0.02 & 0.011 \\
\hline A39 & 2 & 1 & 1 & 1 & 0.187 & & 1 & & 0.071 & & & & 0 & 0.01 & 0.006 \\
\hline A40 & 2.33 & 1 & 2 & 2 & 0.312 & & & & 0 & & & & 0 & 0 & 0 \\
\hline A41 & 2.25 & 1 & 3 & 3 & 0.437 & 2 & & & 0.142 & & & & 0 & 0.06 & 0.027 \\
\hline A42 & 2 & 2 & 3 & & 0.312 & 1 & 1 & & 0.142 & & & & 0 & 0.04 & 0.022 \\
\hline A43 & 1.33 & 1 & 1 & & 0.125 & 2 & & & 0.142 & & & & 0 & 0.02 & 0.013 \\
\hline A44 & 1.16 & 1 & 1 & & 0.125 & 2 & 1 & & 0.021 & & & & 0 & 0.03 & 0.023 \\
\hline A45 & 1 & 2 & & & 0.125 & & 1 & & 0.071 & & & & 0 & 0.01 & 0.008 \\
\hline A46 & 2.5 & 2 & 1 & 2 & 0.312 & & 1 & & 0.071 & & & & 0 & 0.02 & 0.008 \\
\hline A47 & 1.5 & 2 & 2 & 1 & 0.312 & & 1 & & 0.071 & & & & 0 & 0.02 & 0.014 \\
\hline A48 & 1 & & & 1 & 0.062 & & & 1 & 0.071 & & & & 0 & 0 & 0.004 \\
\hline A49 & 1 & & 1 & 1 & 0.125 & & & & 0 & & & & 0 & 0 & 0 \\
\hline A50 & 1.2 & 2 & 3 & & 0.312 & 1 & & & 0.071 & & & & 0 & 0.02 & 0.018 \\
\hline
\end{tabular}




\begin{tabular}{|l|c|c|c|c|c|c|c|c|c|c|c|c|c|c|c|}
\hline TOTAL & 74.21 & 56 & 79 & 29 & 12 & 35 & 29 & 11 & 5.492 & 10 & 20 & 3 & 17 & 1.22 & 14.64 \\
\hline MEDIA & 1.48 & & & & 0.24 & & & & 0.109 & & & & 0.3 & 0.02 & 0.292 \\
\hline
\end{tabular}

\begin{tabular}{|c|c|c|c|c|c|c|c|c|c|c|c|c|c|c|c|}
\hline \multicolumn{16}{|c|}{ Resultados del analisis de correspondencia global postprueba } \\
\hline ALUMNO & DENSIDAD & C.I & C.E & C.A & $\mathrm{CC}$ & R.I & R.E & R.A & CR & $\mathrm{CNCl}$ & CNCE & CNCA & C & q & Q \\
\hline A1 & 1.33 & 2 & & 1 & 0.125 & & & 2 & 0.142 & 1 & & & 0.5 & 0.017 & 0.389 \\
\hline A2 & 1.26 & 3 & 1 & 1 & 0.312 & 1 & 1 & 2 & 0.285 & & 1 & & 0.5 & 0.088 & 0.467 \\
\hline A3 & 1.33 & 1 & 2 & 1 & 0.25 & 1 & 1 & & 0.142 & 1 & & & 0.5 & 0.036 & 0.403 \\
\hline A4 & 1.12 & 2 & 3 & & 0.312 & 1 & 1 & & 0.142 & & 2 & & 1 & 0.044 & 0.87 \\
\hline A5 & 1 & 1 & 2 & 1 & 0.25 & & 1 & & 0.714 & & 1 & & 0.5 & 0.017 & 0.517 \\
\hline A6 & 1 & 1 & 1 & 1 & 0.187 & & 1 & 1 & 0.142 & 1 & & & 0.5 & 0.026 & 0.526 \\
\hline A7 & 1 & & 1 & 2 & 0.187 & & & 1 & 0.071 & & 1 & 1 & 1 & 0.013 & 0.506 \\
\hline A8 & 2 & 2 & 3 & & $\mid 0.312$ & 2 & & 1 & 0.214 & & 1 & & 0.5 & 0.066 & 0.314 \\
\hline A9 & 1.8 & 2 & 2 & & \begin{tabular}{|l|}
0.25 \\
\end{tabular} & & 1 & & 0.071 & 1 & 1 & & 1 & $\mid 0.017$ & \begin{tabular}{|l|}
1.01 \\
\end{tabular} \\
\hline A10 & 1 & 1 & 1 & 1 & 0.187 & & & 2 & 0.142 & & 1 & & 0.5 & 0.085 & 0.39 \\
\hline A11 & 1 & 4 & & & \begin{tabular}{|l|} 
\\
\end{tabular} & 3 & 1 & & 0.285 & 1 & & & 0.5 & 0.712 & 0.571 \\
\hline A12 & 0.714 & 3 & 1 & 1 & 0.312 & & 3 & & 0.214 & 1 & 1 & & 1 & 0.066 & 1.49 \\
\hline A13 & 1.6 & 1 & 2 & & 0.187 & & & 1 & 0.071 & & 1 & & 0.5 & 0.013 & 0.3 \\
\hline A14 & 0.72 & 4 & 1 & 1 & 0.375 & & 3 & & 0.214 & 1 & & 1 & 1 & 0.08 & 1.5 \\
\hline A15 & 1.5 & 3 & & 1 & 0.25 & 1 & 1 & 1 & 0.214 & 1 & & 1 & 1 & 0.535 & 0.702 \\
\hline A16 & 1.2 & 3 & 2 & & 0.312 & & 1 & 2 & 0.214 & & 1 & & 0.5 & 0.066 & 0.472 \\
\hline A17 & 1 & & 2 & 1 & $\mid 0.187$ & & 1 & & 0.071 & & 1 & 1 & 1 & 0.013 & 1.01 \\
\hline A18 & 1.5 & 3 & & & $\mid 0.187$ & 1 & 1 & & 0.142 & 1 & & & 0.5 & 0.026 & 0.351 \\
\hline A19 & 1.13 & 4 & 1 & 1 & 0.375 & 1 & 2 & 1 & 0.285 & 1 & & 1 & 1 & 0.107 & 0.979 \\
\hline A20 & 0.79 & 2 & 4 & 1 & 0.437 & 2 & 1 & 1 & 0.285 & & 2 & & 1 & 0.124 & .1 .42 \\
\hline A21 & 0.91 & 2 & 4 & 1 & 0.437 & 1 & 2 & 2 & 0.357 & & 1 & 1 & 1 & 0.156 & 1.27 \\
\hline A22 & 0.8 & 4 & & & 0.25 & & 3 & & 0.214 & 1 & & & 0.5 & 0.053 & 0.691 \\
\hline A23 & 1 & 4 & 2 & & 0.375 & 2 & 3 & & 0.357 & 1 & & & 0.5 & 0.133 & 0.633 \\
\hline A24 & 1.6 & 2 & 1 & 1 & 0.25 & 1 & & & 0.071 & 1 & & 1 & 1 & 0.017 & 0.636 \\
\hline A25 & 2.23 & 3 & 6 & & 0.562 & 1 & 2 & & 0.214 & 1 & & & 0.5 & 0.12 & 0.278 \\
\hline A26 & 1.05 & 5 & 2 & & $\mid 0.375$ & 3 & 1 & & 0.285 & 2 & & & 1 & 0.107 & 1.05 \\
\hline A27 & 1.12 & 3 & & & \begin{tabular}{|l|}
0.25 \\
\end{tabular} & 1 & 1 & & 0.142 & 2 & & & 1 & 0.035 & 0.924 \\
\hline A28 & 1.5 & 2 & 2 & 1 & 0.187 & & & 2 & 0.142 & 1 & & 1 & 1 & 0.026 & 0.684 \\
\hline A29 & 1.75 & 1 & 5 & 2 & 0.312 & 1 & 1 & & 0.142 & 1 & 1 & & 1 & \begin{tabular}{|l|} 
\\
\end{tabular} .044 & 0.596 \\
\hline A30 & 1.2 & 1 & 2 & & 0.375 & 1 & 1 & 1 & 0.214 & 1 & 1 & & 1 & 0.08 & 0.9 \\
\hline A31 & 1.4 & 4 & & 1 & 0.437 & 2 & 1 & 1 & 0.285 & 1 & & 1 & 1 & 0.124 & 0.803 \\
\hline
\end{tabular}




\begin{tabular}{|c|c|c|c|c|c|c|c|c|c|c|c|c|c|c|c|}
\hline A32 & 2 & 2 & 1 & & 0.125 & & & 1 & 0.071 & 1 & & & 0.5 & 0.009 & 0.254 \\
\hline A33 & 1.37 & 3 & 1 & & 0.312 & 2 & 2 & & 0.357 & 1 & 1 & & 1 & 0.111 & 0.811 \\
\hline A34 & 1 & 3 & 1 & & 0.25 & 2 & 1 & 2 & 0.357 & 2 & & & 1 & 0.089 & 1.089 \\
\hline A35 & 1 & 4 & 1 & & 0.312 & & & 3 & 0.214 & 1 & & & 0.5 & 0.066 & 0.566 \\
\hline A36 & 1.09 & 2 & 2 & & 0.25 & 2 & 2 & & 0.285 & 2 & & & 1 & 0.071 & 0.982 \\
\hline A37 & 1.04 & 6 & 2 & & 0.5 & 2 & 2 & & 0.285 & 2 & & & 1 & 0.142 & 1.09 \\
\hline A38 & 1 & 3 & 3 & & 0.375 & 1 & 1 & & 0.142 & 1 & & & 0.5 & 0.053 & 0.553 \\
\hline A39 & 0.794 & 2 & 2 & 1 & 0.312 & & 1 & 4 & 0.357 & 1 & & & 0.5 & 0.111 & 0.77 \\
\hline A40 & 1.2 & & & & 0.25 & & & & 0.071 & & & & 1 & 0.017 & 0.848 \\
\hline A41 & 1.25 & 2 & 1 & & 0.187 & & & 1 & 0.071 & 1 & & & 0.5 & 0.013 & 0.41 \\
\hline A42 & 1.12 & 1 & 2 & 1 & 0.25 & & & 2 & 0.142 & 1 & 1 & & 1 & 0.035 & 0.92 \\
\hline A43 & 1.13 & 8 & 1 & 2 & 0.687 & 2 & 2 & & 0.285 & 2 & & & 1 & 0.196 & 1.058 \\
\hline A44 & 1.36 & 4 & 2 & & 0.375 & 3 & & 3 & 0.428 & 1 & & & 0.5 & 0.16 & 0.485 \\
\hline A45 & 0.95 & 3 & 2 & 3 & 0.5 & 1 & 2 & 3 & 0.428 & 2 & & & 1 & 0.214 & 1.27 \\
\hline A46 & 1.5 & 3 & 4 & 2 & 0.562 & 3 & 2 & & 0.357 & & 2 & & 1 & 0.2 & 0.8 \\
\hline A47 & 1.12 & 4 & 4 & 1 & 0.5 & 2 & 2 & & 0.285 & 1 & 1 & & 1 & 0.142 & 1.015 \\
\hline A48 & 1.3 & 4 & 4 & 1 & 0.5 & & 1 & 1 & 0.142 & 1 & 1 & & 1 & 0.071 & 0.823 \\
\hline A49 & 1.16 & 3 & 1 & 2 & 0.312 & 1 & & 1 & 0.142 & 1 & & & 0.5 & 0.044 & 0.52 \\
\hline A50 & 1.25 & 5 & 2 & & 0.437 & & 1 & 2 & 0.214 & 2 & & & 1 & 0.093 & 0.874 \\
\hline TOTAL & $\mathbf{5 7 . 4 7 8}$ & $\mathbf{1 2 3}$ & $\mathbf{8 2}$ & $\mathbf{3 0}$ & $\mathbf{1 4 . 8}$ & $\mathbf{4 6}$ & $\mathbf{5 2}$ & $\mathbf{4 0}$ & $\mathbf{1 0 . 6 2}$ & $\mathbf{4 1}$ & $\mathbf{2 2}$ & $\mathbf{9}$ & $\mathbf{3 7}$ & $\mathbf{4 . 6 7 5}$ & $\mathbf{2 1 . 2 3}$ \\
\hline MEDIA & $\mathbf{1 . 2 2}$ & & & & $\mathbf{0 . 3 2 2}$ & & & & $\mathbf{0 . 2 2 2}$ & & & & $\mathbf{0 . 8}$ & $\mathbf{0 . 0 9}$ & $\mathbf{0 . 7 2 7}$ \\
\hline
\end{tabular}

Donde:

CC: Correspondencia conceptual.

C.A.: Conceptos alusivos.

$\mathrm{CR}$; Correspondencia relacional.

R.I.: Relaciones lógicas idénticas

C: Correspondencia en el núcleo conceptual.

R.E.: Relaciones lógicas equivalentes.

q: Calidad de conceptos y relaciones lógicas.

R.A.: Relaciones lógicas alusivas.

Q: Calidad general del discurso.

CNCl: Conceptos del núcleo conceptual idénticos.

C.I.; Conceptos idénticos.

CNCE: Conceptos del núcleo conceptual equivalentes.

C.E.: Conceptos equivalentes.

CNCA: Conceptos del núcleo conceptual alusivos.

\section{Análisis cuantitativo por plantel}

Debido a la heterogeneidad del discurso de los alumnos, no es posible hacer un análisis cualitativo general de éstos, por lo que solo presentamos el análisis cuantitativo tomando como datos las medias obtenidas por plantel . 
Para el CBTis 149 la densidad promedio es $d=1.22$ que se encuentran en el rango de Marco Conceptual. Los valores de correspondencia conceptual, relacional y en el núcleo, en promedio, son los siguientes:
a) $\mathrm{cc}=0.28$
Marco referencial
b) $\mathrm{cr}=0.21$
Marco Nocional
c) $c=0.72$
Marco Conceptual

Como estos valores no se encuentran todos en el mismo rango, el criterio de heterogeneidad en rangos próximos nos ubica esta organización conceptual como un Marco Referencial.

En el caso de el CETis 120 la $d=1.22$ que se encuentra en Marco Conceptual, los valores de correspondencia conceptual, relacional y en el núcleo, promedio, son los siguientes:
a) $\mathrm{Cc}=0.357$
Marco Referencial
b) $\mathrm{cr}=0.233$
Marco Nocional
c) $\mathrm{c}=0.88$
Marco Conceptual

Como estos valores no se encuentran todos en el mismo rango, el criterio de heterogeneidad en rangos próximos nos ubica esta organización conceptual como un Marco Referencial.

Análisis cuantitativo global
a) $\mathrm{Cc}=0.322$
Marco Referencial
b) $\mathrm{cr}=0.222$
Marco Nocional
c) $\mathrm{c}=0.8$
Marco Conceptual

El conjunto de la muestra, en ambos planteles, presenta una calidad general del discurso (Q) que va de 0.292 en la preprueba a 0.727 en la postprueba. Aplicando t de Student a estos datos encontramos una diferencia estadísticamente significativa. Además, se modifica sustancialmente el rango de clasificación de la organización conceptual: pasa de Marco Referencial a Marco Conceptual.

\section{FUENTES CONSULTADAS}

ALUCEMA, M. A., Evaluación de las organizaciones conceptuales de estudiantes de biología referidas al concepto de evolución, en M. A. Campos y R. Ruiz, Problemas de acceso al conocimiento y enseñanza de las ciencias, UNAM, México, 1996.

ALUCEMA, M. A., El discurso áulico y la construcción de conocimiento científico. V Seminario de Epistemología, Cognición y Enseñanza de las Ciencias, UNAM, México, 2001.

AUSUBEL, D., NOVAK, J. D. y HANESIAN, H., Psicología educativa: un punto de vista cognoscitivo, Trillas, México, 1983.

CAMPOS, M. A., CORTÉS, L. y GASPAR, S. Análisis de discurso de la organización lógico-conceptual de estudiantes de Biología de nivel secundaria. Revista Mexicana de Investigación Educativa, Vol. IV (7): 27-77, 1999. 
CAMPOS, M. A. y GASPAR, S., El modelo de análisis proposicional: un método para el estudio de la organización lógico-conceptual del conocimiento, en M. A. Campos y R. Ruiz, Problemas de acceso al conocimiento y enseñanza de las ciencias, México, UNAM, 1996.

CAMPOS, M. A., GASPAR, S. y ALUCEMA, M. A., Análisis de discurso de la conceptualización de estudiantes de Biología en el nivel universitario, Revista Internacional de Ciencias Sociales y Humanidades, Vol. X (1): 31-71, 2000.

CAMPOS, M. A., RUIZ, G. R. y ALUCEMA, M. A., Estructuras conceptuales graduadas en el conocimiento aprendido, en M. A. Campos y R. Ruiz, Problemas de acceso al conocimiento y enseñanza de las ciencias, México, UNAM, 1996.

COLI, C., La construcción de esquemas de conocimiento en situaciones de enseñanza-aprendizaje, en César Coll (Comp.), Psicología genética y aprendizajes escolares, Siglo XXI, Madrid, 1998.

FRAISSE, P. y PIAGET, J., La inteligencia. (Tratado de psicología experimental), Paidos, Buenos Aires, 1973.

MOOL, C. L., Vygotsky y la educación. Connotaciones y aplicaciones de la psicología sociohistórica en la educación, Aique, Buenos Aires, 1993. 


\title{
Contactar
}

Revista lberoamericana de Educación

\author{
Principal OEI
}

